\title{
Enhanced flexural performance of epoxy polymer concrete with short natural fibers
}

\author{
HU Bin ${ }^{1}$, ZHANG NanLi ${ }^{1}$, LIAO YuTian ${ }^{1}$, PAN ZhiWei ${ }^{1}$, LIU YiPing ${ }^{1}$, ZHOU LiCheng $^{1}$, \\ LIU ZeJia ${ }^{1 *}$ \& JIANG ZhenYu ${ }^{1,2^{*}}$ \\ ${ }^{1}$ School of Civil Engineering and Transportation, State Key Laboratory of Subtropical Building Science, South China University of Technology, \\ Guangzhou 510640, China; \\ ${ }^{2}$ The State Key Laboratory of Nonlinear Mechanics, Institute of Mechanics, Chinese Academy of Sciences, Beijing 100190, China
}

Received September 13, 2017; accepted April 28, 2018; published online July 12, 2018

\begin{abstract}
Epoxy polymer concrete (EPC) has found various applications in civil engineering. To enhance the flexural performance of EPC, two kinds of short natural fibers with high specific strength (sisal fibers and ramie fibers) have been incorporated into EPC. The results of mechanical tests show that a small loading of natural fibers ( $0.36 \mathrm{vol} \%)$ can significantly increase the flexural strength of EPC by $25.3 \%$ (ramie fibers) or $10.4 \%$ (sisal fibers). This enhancement is achieved without any sacrifice of compressive strength of EPC. The reinforcing effects of short natural fibers on the flexural properties and compressive properties of EPC decrease with further increase in fiber content, due to the insufficient wetting of fibers by epoxy resin which results in poor interfacial bonding. The reinforcing mechanisms of short natural fibers are explored according to the observation of fracture surfaces and micromechanical modelling. It is found that the parallel model based on the rule of mixture can be a good approximation to describe the improvement in flexural strength of the short natural fiber reinforced EPC at low fiber volume fractions.
\end{abstract}

epoxy polymer concrete (EPC), natural fibers, flexural strength, micromechanics models

Citation: Hu B, Zhang N L, Liao Y T, et al. Enhanced flexural performance of epoxy polymer concrete with short natural fibers. Sci China Tech Sci, 2018, 61, https://doi.org/10.1007/s11431-017-9272-4

\section{Introduction}

Epoxy polymer concrete (EPC) is a type of polymer concrete in which epoxy resin is used as the binder to replace the traditional cement binder [1,2]. Due to its high strength, good corrosion resistance and rapid setting features, EPC is considered as a promising engineering material in construction and has found various applications, e.g. roadway pavement, building cladding, drain components, and machine beds [35]. As Portland cement-based concrete, polymer concrete shows outstanding performance under compression, whereas its tensile strength is relatively poor and sometimes in-

*Corresponding authors (email: zjliu@scut.edu.cn; zhenyujiang@scut.edu.cn) sufficient to applications [6-8]. This vulnerability substantially affects the flexural performance of polymer concrete because failure will occur prematurely in the region subjected to tension. To improve the flexural performance of polymer concrete, short fibers have been incorporated as an additional reinforcing phase. Broniewski et al. [9] found that addition of $3.5 \mathrm{wt} \%$ short steel fibers leads to a significant increase in flexural strength of EPC by about $40 \%$. In the work reported by Vipulanandan et al. [10], addition of $3 \mathrm{wt} \%$ short glass fibers increases the flexural strength of polyester polymer concrete by around $32 \%$. Similar improvement was observed by Griffiths and Ball [11] who dispersed $1.5 \mathrm{wt} \%$ short glass fibers into polyester polymer concrete and attained a $19 \%$ increase in flexural strength. Moreover, the 
flexural performance of EPC with artificial crack, i.e. the fracture toughness of the notched EPC beam under threepoint-bending condition, can be considerably enhanced by the incorporation of short glass fibers [12-14] or short carbon fibers [13].

Natural fiber reinforced composites have attracted growing interest as a kind of eco-friendly engineering material nowadays [15-17]. Compared with synthetic fibers, natural fibers impose much less impact to the environment in both manufacturing stage and recycling stage. Moreover, natural fibers are usually byproduct of agriculture, which makes them a cost-efficient option. In the past decades, various natural fibers have been introduced into traditional cement concrete in order to enhance the tensile and flexural properties of concrete [18-22]. However, the study of natural fiber reinforced polymer concrete remains limited heretofore. Reis et al. [23] incorporated several kinds of natural fibers (i.e. coconut, sugar cane bagasse and banana fibers) into EPC. It was found that coconut fibers contribute positively to the flexural and fracture performance of EPC, sugar cane bagasse fibers only increase the fracture toughness, whereas banana fibers make the EPC weaker. He also tried to add the textile cutting wastes consisting of cotton, polyester, silk and rayon into EPC, but these additives unfortunately result in deterioration of both flexural and compressive performance of EPC [24].

In this paper, short sisal fibers and ramie fibers are incorporated into EPC. The two kinds of natural fibers have the highest specific tensile strengths and Young's moduli among the cellulose-based natural fibers $[25,26]$. The mechanical properties of the natural fiber reinforced EPC (NFREPC) are studied through four-point-bending tests and compression tests. Based on the experimental results, the reinforcing mechanisms are explored combining the observation of fracture surfaces and micromechanical modeling of short fiber reinforced polymer composites.

\section{Sample preparation and testing program}

The raw sisal and ramie continuous fibers are supplied by Yiwu Yangjin Ltd, China. Before being chopped into $10 \mathrm{~mm}-$ long pieces, the two kinds of fibers were soaked in the solution with $1 \%$ sodium hydroxide $(\mathrm{NaOH})$ for 30 min for surface treatment (Figure 1), then washed with water and dried in oven. Table 1 lists the physical and mechanical properties of the two kinds of fibers. The densities were measured using an analytical balance (Mettler Toledo ME 104E) equipped with density determination kits. The diameters are determined from the micrographs acquired by an optical microscope (Olympus BX51M). The strength of the continuous fibers was obtained through the tensile tests on a universal testing machine (Instron 5567).
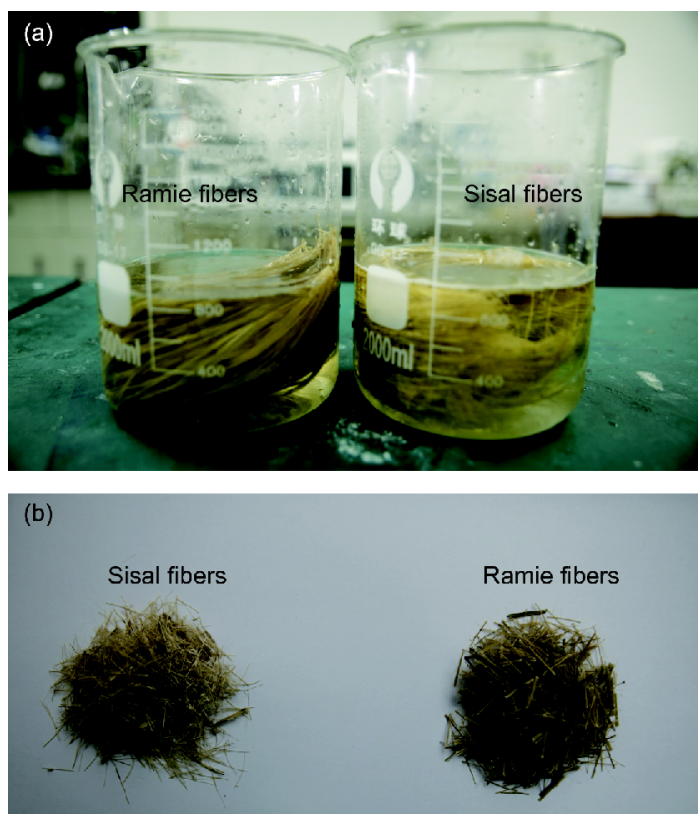

Figure 1 (Color online) (a) Surface treatment of the two kinds of natural fibers; (b) chopped pieces of sisal fibers and ramie fibers.

Table 1 Physical and mechanical properties of sisal fibers and ramie fibers

\begin{tabular}{ccc}
\hline Fiber type & Sisal fiber & Ramie fiber \\
\hline Density $\left(\mathrm{g} \mathrm{cm}^{-3}\right)$ & 1.43 & 1.48 \\
Diameter $(\mathrm{mm})$ & 0.25 & 0.12 \\
Tensile strength $(\mathrm{MPa})$ & 418.77 & 525.97 \\
\hline
\end{tabular}

The granite aggregate is provided by Fhujian Shiyufa Stone Co., Ltd. The weight percentage of the fine aggregate with average size less than $4.75 \mathrm{~mm}$ is about $45 \%$. The weight percentage of the coarse aggregate with average size in the range of $4.75-9.5 \mathrm{~mm}$ is about $55 \%$. The epoxy binder is supplied by Fhuzhou Baisheng Fine Chemicals Pte. Ltd. It is composed of bisphenol-A resin (BS5461A) and amine hardener (BS5462). The Epoxy Equivalent Weight (EEW) and density of the epoxy resin are $200 \mathrm{~g}$ equiv $^{-1}$ and $1.1 \mathrm{~g} \mathrm{~cm}^{-3}$, respectively. The Amine Hydrogen Equivalent Weight (AHEW) and density of the hardener are $100 \mathrm{~g} \mathrm{equiv}^{-1}$ and $0.985 \mathrm{~g} \mathrm{~cm}^{-3}$, respectively.

Prior to the preparation of NFREPC specimens, all the materials are dried in oven at $40^{\circ} \mathrm{C}$ for $4 \mathrm{~h}$. The epoxy resin was first mixed with the hardener following a weight ratio of $2: 1$. Then the short natural fibers were added into the mixture and stirred to disperse uniformly. Afterward, the aggregate was mixed into the epoxy binder. Finally, the mixture was moulded into cuboids $(50 \mathrm{~mm} \times 50 \mathrm{~mm} \times 200 \mathrm{~mm})$ and cylinders $(\phi 100 \mathrm{~mm} \times 100 \mathrm{~mm})$ for bending tests and compression tests, respectively. The NFREPC specimens were cured at $25^{\circ} \mathrm{C}$ for $72 \mathrm{~h}$ before demoulding. In this study, the weight percentage of epoxy matrix is controlled to be $11.5 \mathrm{wt} \%$ (about $22.9 \mathrm{vol} \%$ ). The fiber volume fraction is set in a range 
of $0.12-0.60 \mathrm{vol} \%$. The EPC without natural fibers is used as the control sample.

The flexural properties of the NFREPC are evaluated through the four-point-bending test on an Instron 5567 testing machine at a loading speed of $2 \mathrm{~mm} \mathrm{~min}^{-1}$, as illustrated in Figure 2(a). The flexural strength of concrete specimens is calculated according to

$\sigma_{b}=\frac{P L}{b h^{2}}$

where $P$ is the peak load, $L$ is the span $(L=150 \mathrm{~mm}), b$ is the width of the specimen $(b=50 \mathrm{~mm}), h$ is the height of the specimen $(h=50 \mathrm{~mm})$. The compressive properties of the NFREPC are measured through the axial compression test on a D2-051 2000KN hydraulic pressure testing machine at a loading speed of $2 \mathrm{~mm} \mathrm{~min}^{-1}$, as illustrated in Figure 2(b). For each set of EPC specimens, at least three specimens were tested to eliminate the influence of accidental error.

\section{Results and discussion}

\subsection{Bending and compression tests}

Figure 3 shows the flexural stress-deflection curves of the NFREPC with sisal fibers and ramie fibers, respectively. During the loading process, the bending stress increases almost linearly with the deflection. Then it drops sharply after attaining the peak load, indicating a brittle characteristic of the two kinds of NFREPC. The compressive stress-displacement curves of the two kinds of NFREPC demonstrate the similar behavior.

Tables 2 and 3 list the measured flexural strength and compressive strength of the NFREPC with the two kinds of short natural fibers. It can be seen that a small loading of natural fibers can significantly improve the flexural strength of EPC. The flexural strength of EPC with $0.36 \mathrm{vol} \%$ sisal fibers and ramie fibers increases by $10.4 \%$ and $25.3 \%$ respectively, compared with the control sample. Compared with other natural fibers, such as coconut fibers $(25.1 \%)$ and sugar cane fibers $(3.5 \%)$ [23], sisal fibers and ramie fibers show excellent enhancement effects. This improvement is not at compromise with the compressive strength. The compressive strength of the two kinds of NFREPC increases by $5.9 \%-7.5 \%$ when the fiber volume fraction is $0.36 \mathrm{vol} \%$. Further increase of the fiber volume fraction leads to a decrease in the reinforcing effects of natural fibers. The flexural strength and compressive strength of sisal fiber reinforced EPC become lower than those of the control sample when the fiber volume fraction reaches $0.60 \mathrm{vol} \%$. The strength of the $0.60 \mathrm{vol} \%$ ramie fiber reinforced EPC falls back to the level of control sample.

Figure 4 compares the fracture surface of the bending specimens of the NFREPC with $0.36 \mathrm{vol} \%$ and $0.48 \mathrm{vol} \%$ natural fibers. It can be seen in Figure 4(a) and (b) that the breakage of aggregate, epoxy matrix and natural fibers occur
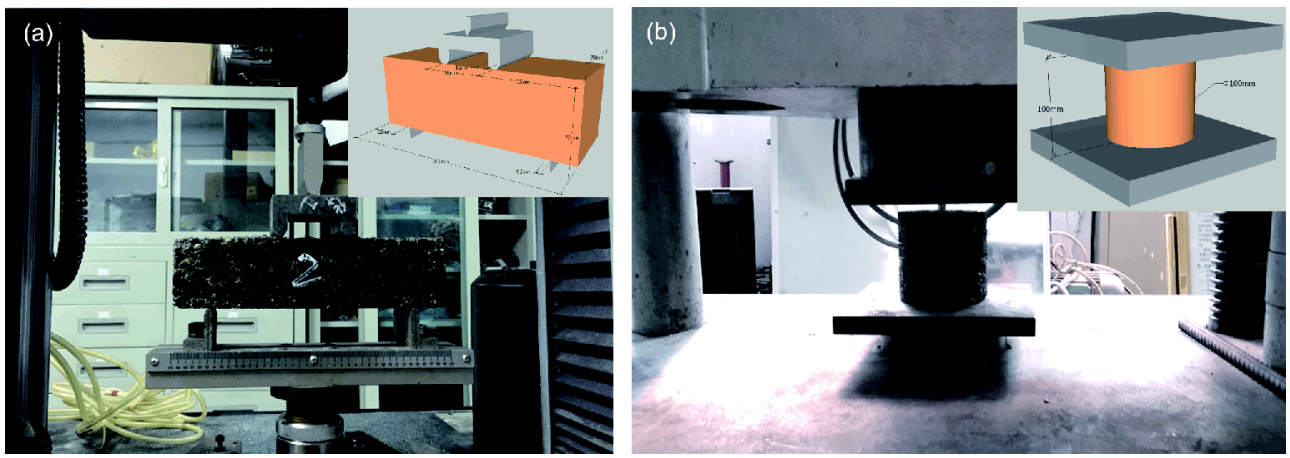

Figure 2 (Color online) Setup of (a) four-point-bending test and (b) compression test for NFREPC.
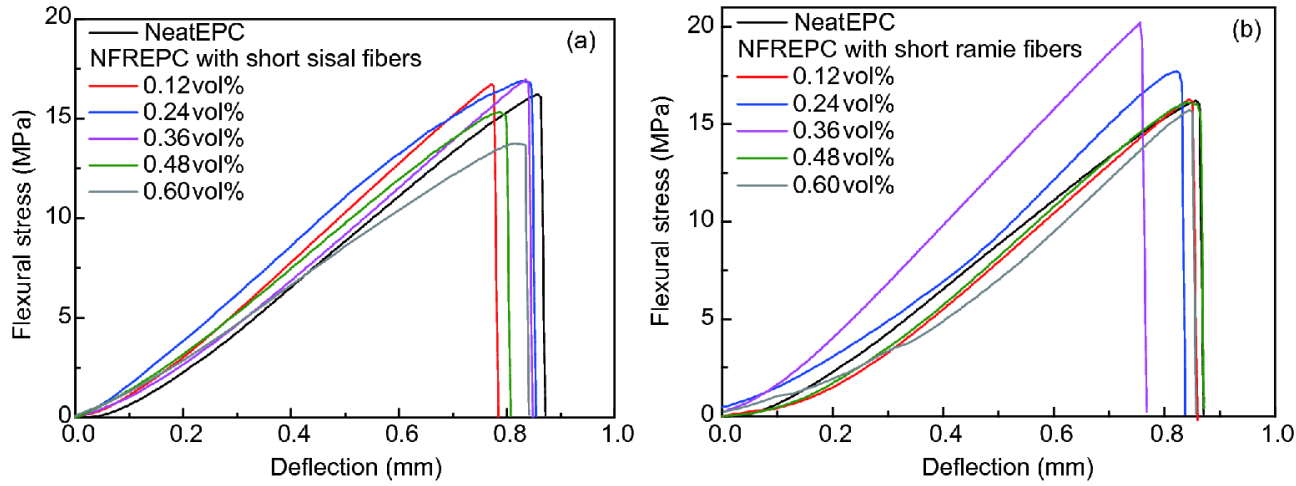

Figure 3 (Color online) Typical flexural stress-deflection curves of the EPC reinforced with (a) short sisal fibers and (b) short ramie fibers. 
Table 2 Flexural strength and compressive strength of the sisal fiber reinforced EPC

\begin{tabular}{ccccc}
\hline Sisal fiber (vol\%) & Flexural strength (MPa) & Coefficient of variation (\%) & Compressive strength (MPa) & Coefficient of variation (\%) \\
\hline 0 & $15.45 \pm 0.77$ & 4.33 & $59.61 \pm 1.21$ & 2.86 \\
0.12 & $16.62 \pm 0.30$ & 1.60 & $58.32 \pm 2.95$ & 4.48 \\
0.24 & $16.78 \pm 0.18$ & 0.47 & N/A & N/A \\
0.36 & $17.05 \pm 0.37$ & 0.80 & $64.08 \pm 1.49$ & 2.07 \\
0.48 & $15.49 \pm 0.29$ & 1.02 & N/A & N/A \\
0.60 & $14.56 \pm 0.81$ & 5.22 & $40.32 \pm 2.63$ & 5.73 \\
\hline
\end{tabular}

Table 3 Flexural strength and compressive strength of the ramie fiber reinforced EPC

\begin{tabular}{ccccc}
\hline Ramie fiber (vol\%) & Flexural strength (MPa) & Coefficient of variation (\%) & Compressive strength (MPa) & Coefficient of variation (\%) \\
\hline 0 & $15.45 \pm 0.77$ & 4.33 & $59.61 \pm 1.21$ & 2.86 \\
0.12 & $17.62 \pm 1.34$ & 6.99 & $60.99 \pm 1.14$ & 1.71 \\
0.24 & $18.25 \pm 0.67$ & 3.21 & N/A & N/A \\
0.36 & $19.36 \pm 0.85$ & 4.37 & $63.15 \pm 2.29$ & 3.15 \\
0.48 & $16.54 \pm 0.42$ & 1.96 & N/A & N/A \\
0.60 & $16.09 \pm 1.04$ & 5.70 & $58.91 \pm 1.44$ & 2.38 \\
\hline
\end{tabular}
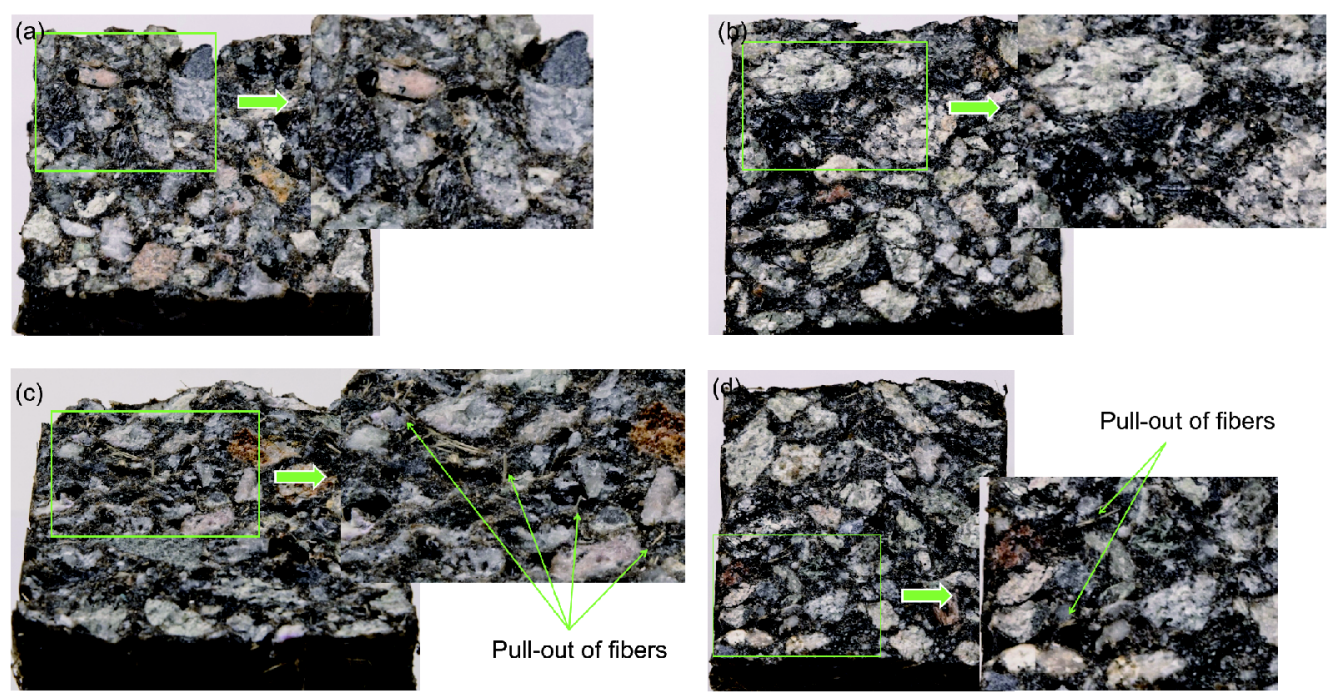

Figure 4 (Color online) Fracture surfaces of the NFREPC with (a) 0.36 vol $\%$ sisal fibers, (b) 0.36 vol\% ramie fibers, (c) 0.48 vol\% sisal fibers and (d) 0.48 vol\% ramie fibers. The exposed fibers on the facture surfaces are marked with circles.

almost simultaneously in the bending specimens with $0.36 \mathrm{vol} \%$ natural fibers, whereas substantial pull-out of fibers are visible on the fracture surface of the NFREPC with $0.48 \mathrm{vol} \%$ natural fibers (Figure $4(\mathrm{c})$ and (d)). This phenomenon indicates that the wetting of fibers could be poor when the fiber volume fraction is at a relatively high level, leading to a weak bonding between the fibers and matrix.

Figure 5 shows the fractured compressive specimens of the NFREPC with 0.36 vol $\%$ natural fibers. The failure behavior of the two kinds of NFREPC is quite similar to the traditional cement concrete. The main cracks propagate in the direction along the compression direction. This fracture mode in- validates the enhancing effects of short fiber because of the poor resistance of natural fiber to shear force.

\subsection{Reinforcing mechanisms}

The reinforcing effect of natural fibers on the flexural strength of EPC can be described by the micromechanics models developed for the composites reinforced with synthetic short fibers. In this study, natural fibers are incorporated at very low loadings, and the fracture of all specimens starts in the tension region during the four-pointbending tests. Thus the Halpin-Tsai equations [27], which 

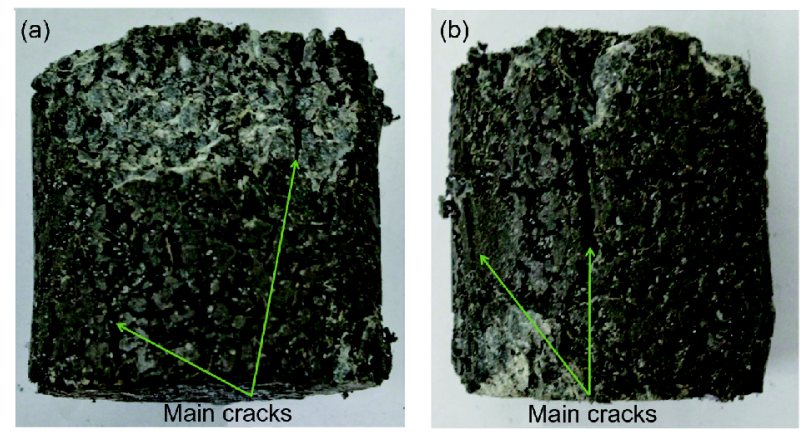

Figure 5 (Color online) Fractured NFREPC specimens under compression with (a) $0.36 \mathrm{vol} \%$ ramie fibers and (b) $0.36 \mathrm{vol} \%$ sisal fibers.

can fit the experimental results well for the short fiber reinforced composites with low fiber volume fractions, are employed to establish the relation between the flexural strength of NFREPC and the tensile strength of natural fibers. Assuming that the natural fibers are uniformly dispersed in the NFREPC with random orientation and a good bonding between the fibers and matrix is achieved, the Halpin-Tsai equations can be used to predict the modulus of composite [28]:

$$
\begin{aligned}
& \frac{E_{c}}{E_{m}}=\frac{1}{5}\left(\frac{1+\frac{2 l}{d} \eta_{L} V_{f}}{1-\eta_{L} V_{f}}\right)+\frac{4}{5}\left(\frac{1+2 \eta_{T} V_{f}}{1-\eta_{T} V_{f}}\right), \\
& \eta_{L}=\frac{\frac{E_{f}}{E_{m}}-1}{\frac{E_{f}}{E_{m}}+\frac{2 l}{d}}, \quad \eta_{T}=\frac{\frac{E_{f}}{E_{m}}-1}{\frac{E_{f}}{E_{m}}+2},
\end{aligned}
$$

where $E_{c}$ represents the modulus of composite. $E_{m}$ and $E_{f}$ are the modulus of polymer matrix and fiber, respectively. $l, d$ and $V_{f}$ are the length, diameter and volume fraction of short fibers, respectively. $\eta_{L}$ and $\eta_{T}$ denote the efficiency coefficients of the fibers in the direction parallel and perpendicular to the tensile load applied on the composite, respectively. It is found in Figure 3 that the flexural stress-deflection curves of NFREPC specimens keep a quasi-linear manner in the fourpoint-bending test before the stress reaches the ultimate strength. Thus, the flexural strength of NFREPC can be described in a similar form of eq. (2):

$$
\begin{aligned}
& \frac{\sigma_{c}}{\sigma_{m}}=\frac{1}{5}\left(\frac{1+\frac{2 l}{d} \eta_{L} V_{f}}{1-\eta_{L} V_{f}}\right)+\frac{4}{5}\left(\frac{1+2 \eta_{T} V_{f}}{1-\eta_{T} V_{f}}\right), \\
& \eta_{L}=\frac{\frac{\sigma_{f}}{\sigma_{m}}-1}{\frac{\sigma_{f}}{\sigma_{m}}+\frac{2 l}{d}}, \quad \eta_{T}=\frac{\frac{\sigma_{f}}{\sigma_{m}}-1}{\frac{\sigma_{f}}{\sigma_{m}}+2},
\end{aligned}
$$

where $\sigma_{c}$ and $\sigma_{m}$ represents the flexural strength of NFREPC and EPC without natural fibers respectively, considering the EPC without natural fibers as approximately homogeneous and isotropic matrix. $\sigma_{f}$ denotes the tensile strength of natural fibers.

Figure 6 compares the flexural strength predicted by eq. (3) and the measured results of the two kinds of NFREPC. The theoretical prediction can reproduce the trend observed in the experimental data, i.e. the flexural strength of NFREPC increases with the fiber volume fraction in a specific range (e.g. $0-0.36 \mathrm{vol} \%$ ). However, the predicted values are considerably lower than the measured ones. The maximum relative deviation is found to be $7.4 \%$ for sisal fiber reinforced EPC and $18.0 \%$ for ramie fiber reinforced EPC, respectively. The possible reason can be attributed to that the natural fibers in the concrete are not randomly oriented. According to the observation of fracture surfaces for the bending specimens (Figure 4(a) and (b)), it can be assumed that most fibers on the fracture surface are in the similar direction of the tensile load. Thus eq. (3) can be reduced to

$$
\frac{\sigma_{c}}{\sigma_{m}}=\frac{1+\frac{2 l}{d} \eta_{L} V_{f}}{1-\eta_{L} V_{f}} .
$$

Since the aspect ratio of the short natural fibers $(l / d)$ used in this study is quite large, ranging from 40 to 83 , eq. (5) can be further simplified to the parallel model based on the rule of mixture [29], i.e.

$\sigma_{c}=\sigma_{f} V_{f}+\sigma_{m}\left(1-V_{f}\right)$.
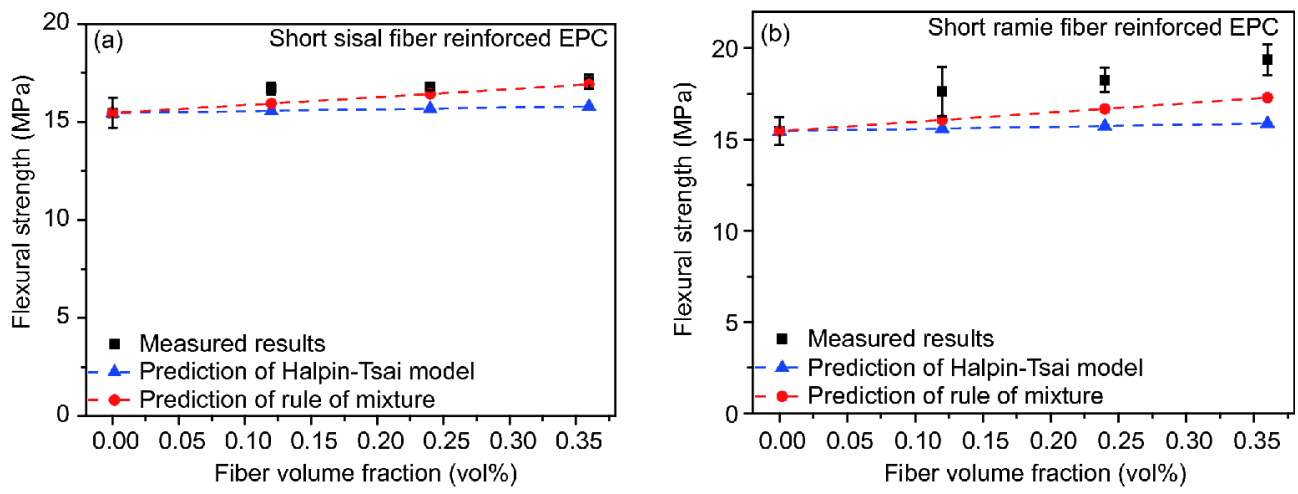

Figure 6 (Color online) Measured flexural strength and the results predicted by the micromechanics models for the NFREPC with (a) short sisal fibers and (b) short ramie fibers. 
It is noteworthy that the premise of eq. (5), i.e. the deformation of aggregate and epoxy matrix keeps consistent, is not rigorously satisfied due to the extraordinary difference in mechanical properties between the aggregate and epoxy matrix. Unlike traditional composites, in which the reinforcements with higher modulus and strength carry more load, the aggregate in EPC has high modulus but low strength whereas the epoxy matrix has low modulus but high strength. In this context, the failure of NFREPC specimens during the bending tests can be considered as a few sequentially occurred events. The aggregate breaks first, then the epoxy matrix with natural fibers takes the whole load and reaches its limit in extremely short time. Figure 3 shows that all the stress-strain curves increase quasi-linearly before a steep drop, without any secondary stress drop related with the failure of aggregate or epoxy matrix with natural fibers. Furthermore, the smooth fracture surfaces in Figure 4(a) and (b) indicate that the epoxy matrix with natural fibers does not experience discernible further deformation after the breakage of the aggregate. Therefore, eq. (5) can be regarded as a close approximation to the situation.

The flexural strength of sisal fiber reinforced EPC predicted by eq. (5) agrees well with the measured results (Figure 6(a)), indicating that the parallel model based on the rule of mixture is a good approximation for the sisal fiber reinforced EPC. The predicted strengths of ramie fiber reinforced EPC are closer to the measured results, compared with prediction given by eq. (3), as shown in Figure 6(b). The maximum relative deviation is reduced from $18.0 \%$ to $10.7 \%$, which can be regarded as an acceptable level. The discrepancy between the predicted results and measured results in ramie fiber reinforced EPC may be associated with the characteristics of ramie fibers. It is found that a piece of as-received individual ramie fiber is a bundle of fine fibers with relatively weak adhesion. During the tension test of individual ramie fiber, the fine fibers are observed to detach from the main bundle and break early, which makes the measured tensile strength of ramie fiber scatter in a wide range. Consequently, a simply picked mean value may lead to considerable underestimation of fiber strength and the strength of composites.

The failure of EPC under compression mainly follows split mode. In this situation, the load-bear capacity of natural fibers subjected to shear is much less than their tensile strength. Therefore, the addition of natural fibers has insignificant effect on the compressive strength of EPC unless the insufficient wetting of fibers occurs at relatively high fiber volume fractions.

\section{Conclusions}

The reinforcing effects of short sisal fibers and ramie fibers on the flexural performance of epoxy polymer concrete have been studied through four-point bending tests. It is found that the two kinds of natural fibers with small volume fraction $(0.12-0.36 \mathrm{vol} \%)$ can lead to a significant increase in the flexural strength of EPC. Ramie fibers demonstrate better reinforcing efficiency than sisal fibers. Incorporation of 0.36 vol\% ramie fibers into EPC results in a $25.3 \%$ increase in the flexural strength, compared with $10.4 \%$ increase by $0.36 \mathrm{vol}$ $\%$ sisal fibers. In addition, this improvement in flexural performance is achieved without sacrifice of the compressive properties. The compressive strength of the two kinds of NFREPC increases slightly with the addition of short natural fibers. However, the reinforcing effects of short natural fibers decay with further increase of fiber content. At the fiber volume fractions higher than 0.36 vol\%, the flexural properties of NFREPC begin decreasing. Both flexural and compressive strength of NFREPC deteriorate to a level even below those of neat EPC when the fiber volume fraction reaches $0.60 \mathrm{vol} \%$. According to the observation of fracture surfaces, this reduction of reinforcing effects is ascribed to the insufficient wetting of fibers by epoxy resin, which causes a poor bonding between the fibers and epoxy matrix.

The reinforcing mechanisms of the natural fibers are explored by introducing the micromechanics models developed for the polymer composites reinforced with short fibers. The discrepancy between the measured results and the values predicted by the Halpin-Tsai model suggests that the short natural fibers works as the reinforcement is not randomly oriented in the EPC. The orientation of natural fibers may be more like the one parallel to the tensile load on the crosssection of bending NFREPC specimen, which makes the prediction of the parallel model based on the rule of mixture attain a good agreement with the experimental data. The availability of the micromechanics model in this situation also indicates a strong fiber-matrix bonding achieved at low fiber volume fractions.

Sisal fibers and ramie fibers are agricultural products of low price. The incorporation of the two kinds of short natural fibers into EPC requires small modification of the procedure of EPC manufacturing. This cost and processing efficiency endows NFREPC with high possibility of large scale use in engineering. For instance, the pavement of highways and bridges is subjected to compression load and bending load simultaneously under its service condition. It could be one of the potential applications of the EPC reinforced with sisal fibers and ramie fibers.

This work was supported by the National Natural Science Foundation of China (Grant Nos. 11772131 \& 11772132), the Natural Science Foundation of Guangdong Province, China (Grant Nos. 2015 A030311046 \& 2015B010131009), the Opening Fund of State Key Laboratory of Nonlinear Mechanics (LNM), CAS, and South China University of Technology (Grant No. 2017ZD096). The authors would thank Prof. Chun Wei (Guilin University of Technology) for the inspiring discussions. 
1 Davydov S S, Solomatov V I, Shvidko Y I. Epoxy polymer concrete. Hydrotech Construct, 1970, 4: 849-852

2 Abdel-Fattah H, El-Hawary M M. Flexural behavior of polymer concrete. Constr Build Mater, 1999, 13: 253-262

3 Ohama Y. Recent progress in concrete-polymer composites. Adv Cement Based Mater, 1997, 5: 31-40

4 Fowler D W. Polymers in concrete: A vision for the 21st century. Cement Concrete Composites, 1999, 21: 449-452

5 Bedi R, Chandra R, Singh S P. Mechanical properties of polymer concrete. J Compos, 2013, 2013: 1-12

6 Etmanski B, Bledzki A K. Glass fiber profiles as reinforcing material in highly filled epoxy polymer concrete. Polymer-Plastics Tech Eng, 1993, 32: 385-396

7 Ribeiro M C S, Tavares C M L, Figueiredo M, et al. Bending characteristics of resin concretes. Mat Res, 2003, 6: 247-254

8 Uygunoğlu T, Brostow $\mathrm{W}$, Gencel $\mathrm{O}$, et al. Bond strength of polymer lightweight aggregate concrete. Polym Compos, 2013, 34: 2125-2132

9 Broniewski T, Jamrozy Z, Kapko J. Long life strength polymer concrete. In: Proceedings of the 1st International Congress on Polymer Concretes-Polymers in Concrete, 1976

10 Vipulanandan C, Dharmarajan N, Ching E. Mechanical behaviour of polymer concrete systems. Mater Struct, 1988, 21: 268-277

11 Griffiths R, Ball A. An assessment of the properties and degradation behaviour of glass-fibre-reinforced polyester polymer concrete. Compos Sci Technol, 2000, 60: 2747-2753

12 Reis J M L, de Oliveira R, Ferreira A J M, et al. A NDT assessment of fracture mechanics properties of fiber reinforced polymer concrete. Polymer Testing, 2003, 22: 395-401

13 Reis J M L, Ferreira A J M. Assessment of fracture properties of epoxy polymer concrete reinforced with short carbon and glass fibers. Constr Build Mater, 2004, 18: 523-528

14 Heidari-Rarani M, Aliha M R M, Shokrieh M M, et al. Mechanical durability of an optimized polymer concrete under various thermal cyclic loadings-An experimental study. Constr Build Mater, 2014, 64: $308-315$
15 Bledzki A, Gassan J. Composites reinforced with cellulose based fibres. Prog Polymer Sci, 1999, 24: 221-274

16 Bogoeva-Gaceva G, Avella M, Malinconico M, et al. Natural fiber eco-composites. Polym Compos, 2007, 28: 98-107

17 Faruk O, Bledzki A K, Fink H P, et al. Progress report on natural fiber reinforced composites. Macromol Mater Eng, 2014, 299: 9-26

18 Al-Oraimi S K, Seibi A C. Mechanical characterisation and impact behaviour of concrete reinforced with natural fibres. Compos Struct, 1995, 32: 165-171

19 Tolêdo Filho R D, Ghavami K, England G L, et al. Development of vegetable fibre-mortar composites of improved durability. Cement Concrete Composites, 2003, 25: 185-196

20 Ramakrishna G, Sundararajan T. Studies on the durability of natural fibres and the effect of corroded fibres on the strength of mortar. Cement Concrete Compos, 2005, 27: 575-582

21 Li Z, Wang X, Wang L. Properties of hemp fibre reinforced concrete composites. Compos Part A-Appl Sci Manuf, 2006, 37: 497-505

22 Pacheco-Torgal F, Jalali S. Cementitious building materials reinforced with vegetable fibres: A review. Constr Build Mater, 2011, 25: 575581

23 Reis J M L. Fracture and flexural characterization of natural fiberreinforced polymer concrete. Constr Build Mater, 2006, 20: 673-678

24 Reis J M L. Effect of textile waste on the mechanical properties of polymer concrete. Mat Res, 2009, 12: 63-67

25 Li Y, Mai Y W, Ye L. Sisal fibre and its composites: A review of recent developments. Compos Sci Technol, 2000, 60: 2037-2055

26 Pickering K L, Efendy M G A, Le T M. A review of recent developments in natural fibre composites and their mechanical performance. Compos Part A-Appl S, 2016, 83: 98-112

27 Affdl J C H, Kardos J L. The Halpin-Tsai equations: A review. Polym Eng Sci, 1976, 16: 344-352

28 Lavengood R E, Goettler L A. Stiffness of non-aligned fiber reinforced composites. US Government R\&D Reports, 1971

29 Mallick P K. Fiber-Reinforced Composites: Materials, Manufacturing, and Design. Boca Raton: CRC Press, 2007 